\title{
Amplitude and size scaling for interchange motions of plasma filaments
}

\author{
R. Kubt* and O. E. Garcia \\ Department of Physics and Technology, UiT The Arctic University of Norway, N-9037 Tromsø, Norway \\ M. Wiesenberger \\ Institute for Ion Physics and Applied Physics, Universität Innsbruck, A-6020 Innsbruck, Austria
}

(Dated: March 29, 2018)

\begin{abstract}
The interchange dynamics and velocity scaling of blob-like plasma filaments are investigated using a two-field reduced fluid model. For incompressible flows due to buoyancy the maximum velocity is proportional to the square root of the relative amplitude and the square root of its cross-field size. For compressible flows in a non-uniform magnetic field this square root scaling only holds for ratios of amplitudes to cross-field sizes above a certain threshold value. For small amplitudes and large sizes, the maximum velocity is proportional to the filament amplitude. The acceleration is proportional to the amplitude and independent of the cross-field size in all regimes. This is demonstrated by means of numerical simulations and explained by the energy integrals satisfied by the model.
\end{abstract}

\section{INTRODUCTION}

At the outboard mid-plane of magnetically confined plasmas one universally observes radial motion of fieldaligned plasma pressure perturbations. These are structures of excess pressure localized in the plane perpendicular to the magnetic field and are therefore commonly referred to as blobs [1 and 2]. These blobs are intermittently created close to the last closed magnetic flux surface $[3-6]$ and propagate radially outwards through the scrape-off layer, mediating a significant loss channel for particles and heat. They may further be responsible for high rates of plasma particle recycling at the main chamber wall 7 and 8 . Experimental studies suggest that their dynamical properties set the profile length scale of the particle density profile in the scrape-off layer 9 11]. A stochastic model, which models density fluctuation time series in the scrape-off layer as the superposition of exponentially decaying pulses, explicitly relates the scale length of the density profile to the average radial blob velocity [12].

A large body of research suggests that interchange motions due to the non-uniform magnetic field is the mechanism underlying blob propagation [13 18]. At the outboard mid plane of a magnetically confined plasma magnetic gradient and curvature drifts guide electrons and ions in opposing directions. As a consequence, a blob of excess pressure will be electrically polarized, generating a dipolar potential structure that is out of phase with the pressure perturbation. The resulting electric drift propagates the plasma blob radially outwards, away from the closed field line region, thereby exchanging hot and dense with cold, low density plasma [16 20]. Analysis of data time series shows that the observed motion of the plasma blobs agrees well with the suggested theory [3, 11, 21 24].

Plasma filaments have also been investigated in basic toroidal plasma experiments. In cold plasma experiments

\footnotetext{
* E-mail: ralph.kube@uit.no
}

performed at the Versatile Toroidal Facility it was observed that plasma blobs develop a mushroom shape, as often observed in numerical simulations, and that their flow field is dipolar [25]. Experiments performed in an open field line configuration at the TORPEX device further corroborate that the interchange mechanism supports blob propagation [26 28].

In the equatorial F-layer ionosphere, so-called equatorial spread-F plasma depletions, or bubbles, have been observed to propagate radially out across the boundary of the F-layer ionosphere [29]. Recent measurements of magnetospherical plasmas at Saturn [30] and analytic theory [31] suggest that plasma bubbles propagating through plasma sheets in these regions are also driven by buoyancy.

The simplest fluid models used to describe the interchange dynamics of seeded plasma blobs feature the advection of the particle density by the electric drift 15, 16, 32, and 33. For self-consistent dynamics the electric potential is computed by invoking quasi-neutrality in the low-frequency limit, often applying the so-called Boussinesq approximation. Within this approximation, which is valid for small particle density perturbations $\widetilde{n}$ relative to the background $N,|\widetilde{n}| / N \ll 1$, one neglects particle density gradients in the inertial terms of the fluid equations while retaining them in the other terms. Recent work avoids this simplification [34 39].

In previous works a scale analysis of the model equations was employed to derive velocity scaling laws for blobs, 15, 26, 40 42]. In the simplest case only particle density fluctuations on a uniform background are considered and the parallel dynamics are neglected. Scale analysis of this model suggests that the radial center of mass velocity of blobs follows the so-called inertial scaling $V \sim\left(\ell \triangle n / R_{0} N\right)^{1 / 2}[15]$. Here, $\ell$ is the initial blob cross-field size, $R_{0}$ is the major radius, and $\triangle n$ the initial blob amplitude.

Numerical simulations of incompressible fluid models recover the inertial velocity scaling in the case of small initial blob amplitudes [41]. Numerical simulations relaxing the Boussinesq approximation further validate the 
inertial velocity scaling for blobs with moderate initial amplitudes 35 37]. First indications that the inertial velocity scaling does not hold for blob motions with small amplitudes using a model with compressible flows were reported in [38 and 43].

In this contribution we review the simplifications leading to the reduced two-field fluid models and discuss the common practice of neglecting drift compression terms in the particle continuity equation. This is often justified due to the smallness of the terms and has been applied throughout the literature [14, 15, 19, 32 35, 41, 50 52. We derive conservation laws from the model equations and discuss how neglecting drift compression terms impacts the conservation properties of the model equations. Velocity scaling laws are derived from the conservation laws and compared to the velocity scaling usually derived from the vorticity equation. Numerical simulations of seeded blob propagation are performed with and without drift compression terms in the continuity equation, using two different sets of numerical methods. The resulting velocity scaling of the seeded plasma blobs is compared to analytically derived scaling laws.

\section{ANALYTIC MODELING}

For an isothermal, quasi-neutral plasma with a single cold ion species, the low-frequency electrostatic dynamics is described by the continuity equation for the plasma particle density $n$,

$$
\frac{\partial n}{\partial t}+\nabla \cdot\left(n \boldsymbol{u}_{\mathrm{E}}+n \boldsymbol{u}_{\mathrm{de}}\right)=\nu \nabla_{\perp}^{2} n .
$$

Here, the electric drift is given by $\boldsymbol{u}_{\mathrm{E}}=\boldsymbol{b} \times \nabla \phi / B$ and the diamagnetic drift by $\boldsymbol{u}_{\mathrm{de}}=-\left(T_{\mathrm{e}} /\right.$ en $\left.B\right) \boldsymbol{b} \times \nabla n$, where $\phi$ is the electric potential, $\boldsymbol{B}=B \boldsymbol{b}$ the magnetic field, $e$ the elementary charge and $T_{\mathrm{e}}$ the electron temperature. We consider a slab magnetic field given by $\boldsymbol{B}=\left(B_{0} R_{0} / x\right) \boldsymbol{e}_{z}$ in a Cartesian coordinate system. This field approximates the magnetic field at the outboard mid plane using the radial coordinate $x$, the approximately poloidal coordinate $y$ and the $z$ direction aligned to the magnetic field. We further assume that the aspect ratio $R_{0} / a$, where $R_{0}$ is the major and $a$ is the minor radius, is small as to approximate $1 / B \approx 1 / B_{0}$ within the bounds of the model. In this approximation the curvature of the magnetic field vanishes, $(\boldsymbol{b} \cdot \nabla) \boldsymbol{b}=\mathbf{0}$. Introducing the operator $\mathcal{K}(u) \equiv$ $\nabla \cdot(\boldsymbol{b} \times \nabla u / B)=-\left(1 / B_{0} R_{0}\right) \partial u / \partial y$ allows us to write the drift compression terms as $\nabla \cdot\left(n \boldsymbol{u}_{\mathrm{de}}\right)=\left(T_{\mathrm{e}} / e\right) \mathcal{K}(n)$ and $\nabla \cdot \boldsymbol{u}_{\mathrm{E}}=-\mathcal{K}(\phi)$, respectively.

The particle density is now separated into a stationary and homogeneous background $N$ and a perturbation $\widetilde{n}$ as $n=N+\widetilde{n}$, where we assume that the relative perturbation amplitude is small, $|\widetilde{n}| / N \ll 1$. An energetically consistent model that describes the dynamics of density perturbations in the limit of the Boussinesq approxima- tion is then given by

$$
\begin{aligned}
& \left(\frac{\partial}{\partial t}+\frac{\boldsymbol{b} \times \nabla \phi}{B_{0}} \cdot \nabla\right) \frac{\widetilde{n}}{N}+\alpha \mathcal{K}(\phi)-\beta \frac{T_{\mathrm{e}}}{e} \mathcal{K}\left(\frac{\widetilde{n}}{N}\right)=\nu \nabla_{\perp}^{2} \frac{\widetilde{n}}{N} \\
& \left(\frac{\partial}{\partial t}+\frac{\boldsymbol{b} \times \nabla \phi}{B_{0}} \cdot \nabla\right) \Omega-\Omega_{\mathrm{ci}} \frac{T_{\mathrm{e}}}{e} \mathcal{K}(\widetilde{n})=\nu \nabla_{\perp}^{2} \Omega .
\end{aligned}
$$

Here we have introduced the field-aligned vorticity density of the electric drift $\Omega=N \nabla_{\perp}^{2} \phi / B_{0} \approx N \boldsymbol{b} \cdot \nabla \times \boldsymbol{u}_{\mathrm{E}}$ and the ion cyclotron frequency $\Omega_{\mathrm{ci}}=e B_{0} / m_{\mathrm{i}}$. Artificial coefficients $\alpha, \beta \in\{0,1\}$ in front of the drift compression terms in Eq. $2 \mathrm{a}$ allow to isolate the contribution of these terms on the blob dynamics. Choosing $\alpha=\beta=0$ describes a plasma in a homogeneous magnetic field experiencing a gravitational drift with $g=-T_{\mathrm{e}} /\left(R_{0} m_{\mathrm{i}}\right) \equiv$ $-C_{\mathrm{s}}^{2} / R_{0}$ as the gravity. This may be used to describe astrophysical plasmas, specifically ionospherical irregularities such as equatorial spread $\mathrm{F}$ phenomena, which are thought to be caused by the interchange instability 29 and 44]. The model with $\alpha=\beta=1$ also arises when taking the long wavelength limit of a delta-f gyrofluid model 38] and describes compressible electrostatic motions in a non-uniform magnetic field.

To study the evolution of seeded blobs, the density field is initialized as a Gaussian function with no initial vorticity as

$$
\begin{aligned}
& \tilde{n}(\boldsymbol{x}, t=0)=\triangle n \exp \left(-\frac{\boldsymbol{x}^{2}}{2 \ell^{2}}\right), \\
& \Omega(\boldsymbol{x}, t=0)=0 .
\end{aligned}
$$

The initial perturbation amplitude of the blob is given by $\triangle n$ and $\ell$ is its characteristic cross-field size. To obtain conservation laws of Eq. 2 we multiply Eq. 2a with $-N T_{\mathrm{e}} x / R_{0}$ and $\tilde{n} T_{\mathrm{e}}$ respectively and Eq. $2 \mathrm{~b}$ with $-e \phi / \Omega_{c i}$ and integrate the resulting equations over the domain [20]. Adding the results yields two conservation laws

$$
\begin{aligned}
\frac{\mathrm{d}}{\mathrm{d} t}(G+E) & =\Lambda_{G}-\Lambda_{E}, \\
\frac{\mathrm{d}}{\mathrm{d} t}(S+\alpha E) & =-\left(\Lambda_{S}+\alpha \Lambda_{E}\right),
\end{aligned}
$$

with the energy integrals defined as

$$
\begin{aligned}
G(t) & :=m_{\mathrm{i}} g \int \mathrm{d} A \tilde{n} x \\
E(t) & :=\frac{1}{2} m_{\mathrm{i}} N \int \mathrm{d} A\left(\frac{\nabla_{\perp} \phi}{B_{0}}\right)^{2}, \\
S(t) & :=\frac{1}{2} N T_{\mathrm{e}} \int \mathrm{d} A\left(\frac{\tilde{n}}{N}\right)^{2} .
\end{aligned}
$$

They correspond to the potential energy $G$ of the plasma in its effective gravity field, the kinetic energy $E$ and an entropy-like quantity $S$. For the partial integration over the spatial domain we assume boundary terms to 
vanish. The energy dissipation is expressed by $\Lambda_{G}:=$ $-\nu \int \mathrm{d} A T_{\mathrm{e}} x \nabla_{\perp}^{2} n / R_{0}, \Lambda_{E}:=\nu \int \mathrm{d} A m_{\mathrm{i}} \Omega^{2} / N$ and $\Lambda_{S}:=$ $\nu \int \mathrm{d} A T_{\mathrm{e}}\left(\nabla_{\perp} \tilde{n}\right)^{2} / N$. Then Eq. 4a may be interpreted as the evolution of the non-linearly conserved energy of the system while Eq. $4 \mathrm{~b}$ expresses non-linear conservation of a free-energy like quantity. With Eqs. 3, initial conditions on Eqs. 5 are given by $S(0)=N T_{\mathrm{e}} \pi \ell^{2}(\triangle n / N)^{2} / 2$ and $G(0)=E(0)=0$.

The transfer between potential and kinetic energy, as well as between kinetic energy and the entropy is mediated by the coupling term $\mathrm{d} E / \mathrm{d} t=-\mathrm{d} G / \mathrm{d} t=$ $\int \mathrm{d} A \tilde{n} T_{\mathrm{e}} \mathcal{K}(\phi)$, where we neglect diffusion. It describes a transfer of potential energy of a plasma structure in an effective gravitational field into kinetic energy. Including compression of the electric drift, $\alpha=1$, this coupling term mediates a transfer of $S$ into the kinetic energy of the system [45]. While Eq. 4a sets no bound on either $G$ or $E$, Eq. $4 \mathrm{~b}$ is a restriction for $E$ since both $S \geq 0$ and $E \geq 0$. In other words, the compression of the electric drift introduces an upper bound on the kinetic energy through conservation of internal energy as described by Eqs. 4.

To obtain a velocity scaling of seeded plasma blobs we perform an order of magnitude estimate by assuming that the dynamics of the flow is due to inertia $\partial / \partial t \sim$ $\Omega \sim \phi / B \ell^{2}$, as well as $(1 / N) \partial n / \partial y \sim \triangle n /(N \ell)$ in Eq. $2 \mathrm{~b}$ to obtain [15]

$$
\frac{\max V}{C_{\mathrm{s}}}=\mathcal{R}\left(\frac{\ell}{R_{0}} \frac{\triangle n}{N}\right)^{1 / 2} .
$$

Here, $\mathcal{R}$ is a proportionality factor that will later be determined from numerical simulations. This scaling is valid for both compressible and incompressible flows and is often called the inertial scaling [15]. In this regime, the velocity is proportional to the square root of the blobs cross-field size $\ell$ and its relative perturbation amplitude $\triangle n / N$.

To obtain a velocity scaling law from the conservation laws given by Eqs. 4 we introduce the radial center of mass coordinate of a plasma blob [15]

$$
X(t)=\frac{\int \mathrm{d} A n x}{M},
$$

where $M=\int \mathrm{d} A \tilde{n}=2 \pi \ell^{2} \triangle n$ is the conserved mass. Using Eqs. 2 the radial center of mass velocity $V(t)=$ $\mathrm{d} X / \mathrm{d} t$ can be written as [19]

$$
V(t)=-\frac{1}{M} \int \mathrm{d} A \tilde{n} \frac{1}{B_{0}} \frac{\partial \phi}{\partial y} .
$$

The conservation law given by Eq. $4 \mathrm{~b}$ yields bounds on the entropy and energy as $S(t) \leq S(0)$ and $E(t) \leq S(0)$. An upper bound on the center of mass velocity given by Eq. 8 is then found by applying the Cauchy-Schwarz inequality as

$$
\begin{aligned}
(M V)^{2} & =\left(\int \mathrm{d} A \tilde{n} \frac{1}{B_{0}} \frac{\partial \phi}{\partial y}\right)^{2} \\
& \leq \int \mathrm{d} A \tilde{n}^{2} \int \mathrm{d} A\left(\frac{\nabla_{\perp} \phi}{B_{0}}\right)^{2} \\
& \leq \frac{4}{m_{\mathrm{i}} T_{\mathrm{e}}} S^{2}(0) .
\end{aligned}
$$

This shows that the center of mass velocity is bounded by the initial entropy of the plasma. This initial entropy on the other hand is set by Eq. 3a such that a blobs initial amplitude may set an upper limit on its maximal radial velocity.

Using the initial conditions on Eqs. 5 we evaluate this upper bound on the center of mass velocity as

$$
\frac{\max V}{C_{\mathrm{s}}}=\frac{\mathcal{P}}{4} \frac{\triangle n}{N} .
$$

Here $\mathcal{P}$ is a numerical coefficient with $0<\mathcal{P} \leq 1$ that will later be determined from numerical simulations. This upper bound on the center of mass velocity is a direct consequence of energy conservation and must hold at any stage of the blobs evolution in the case of a non-uniform magnetic field.

We equate Eqs. 6 and 10 to evaluate the critical ratio of initial amplitude to size, above which the velocity is constrained by Eq. 6 rather than the linear scaling given by Eq. 10 as

$$
\left(\frac{\triangle n / N}{\ell / R_{0}}\right)_{\mathrm{c}}=\left(\frac{4 \mathcal{R}}{\mathcal{P}}\right)^{2}
$$

In the case of a non-uniform magnetic field we thus expect large amplitude blobs with small cross-field sizes to be subject to the inertial velocity scaling given by Eq. 6.

To find a scaling for the center of mass acceleration we rewrite Eq. 9 as

$$
(M V)^{2} \leq \frac{4 S(0)}{m_{\mathrm{i}} T_{\mathrm{e}}} E(t),
$$

which is true for both the compressible and the incompressible case. Further assuming that the blob accelerates uniformly in the initial phase, $15,19,33,37,38$, and 41], $V=A t$ and $X=A t^{2} / 2$, and using Eq. 4a, the time derivative yields

$$
A=\frac{\mathcal{Q}}{2} \frac{\triangle n}{N} \frac{C_{\mathrm{s}}^{2}}{R_{0}} \equiv \frac{\mathcal{Q}}{2} g \frac{\triangle n}{N},
$$

where again $\mathcal{Q}$ is a numerical coefficient with $0<\mathcal{Q} \leq 1$. This shows that the blobs is always accelerated with a rate given by the effective gravity $C_{\mathrm{s}}^{2} / R_{0}$ and its initial perturbation amplitude. Such a uniform acceleration is in accordance with previous work where a scale analysis suggests that the temporal scale of the interchange motions described by Eq. $2 \mathrm{~b}$ is given by $\gamma=(g \triangle n / \ell N)^{1 / 2}$ [15. 
Finally, we introduce the time it takes a blob to accelerate to its maximal velocity as $t_{\max V}$. Using $\max V=$ $t_{\max V} A$ we evaluate this time to be

$$
\frac{t_{\max V}}{R_{0} / C_{\mathrm{s}}}=\frac{2 \mathcal{R}}{\mathcal{Q}} \begin{cases}\frac{\mathcal{P}}{4 \mathcal{R}} & \text { for } \frac{\ell / R_{0}}{\triangle n / N}>\left(\frac{\mathcal{P}}{4 \mathcal{R}}\right)^{2} \\ \sqrt{\frac{\ell / R_{0}}{\triangle n / N}} & \text { otherwise }\end{cases}
$$

Thus, as a consequence of the constant acceleration phase, the time it takes the blob to achieve its maximal radial velocity is independent of its initial amplitude or size when the amplitude is small and the size is large. In the opposite regime, large amplitude blobs feature a shorter acceleration phase than small amplitude blobs and vice versa for the blob size.

\section{NUMERICAL SIMULATIONS}

We continue by investigating the effect of the drift compression terms $\nabla \cdot\left(n \boldsymbol{u}_{\mathrm{de}}\right)$ and $n \nabla \cdot \boldsymbol{u}_{\mathrm{E}}$ in Eq. 2a on the center of mass dynamics of seeded plasma blobs by numerical simulations. To this end we normalize the spatial scales to $\ell$, the temporal scale to the interchange rate $\gamma=\left(C_{\mathrm{s}}^{2} /\left(R_{0} \ell\right)\right)^{1 / 2}$, the electric potential as $\phi \rightarrow \widehat{\phi}=\phi /\left(\gamma B_{0} \ell^{2}\right)$, and the vorticity density as $\Omega \rightarrow \widehat{\Omega}=\Omega / N \gamma$ to rewrite Eqs. 22 in dimensionless form

$$
\begin{aligned}
\frac{\partial n}{\partial t}+\{\phi, n\}-\alpha \kappa \frac{\partial \phi}{\partial y}+\beta \delta \frac{\partial n}{\partial y} & =\nu \nabla_{\perp}^{2} n, \\
\frac{\partial \Omega}{\partial t}+\{\phi, \Omega\}+\frac{\partial n}{\partial y} & =\nu \nabla_{\perp}^{2} \Omega .
\end{aligned}
$$

The free parameters of this model are $\kappa \equiv \ell / R_{0}$, which sets the cross-field size of the plasma blob as a fraction of the major radius and $\delta \equiv \gamma / \Omega_{\mathrm{ci}} \equiv \rho_{s} /\left(R_{0} \ell\right)^{1 / 2}$. This parameter gives the ratio of the interchange time scale relative to the ion cyclotron frequency, or alternatively, the ratio of the thermal gyroradius $\rho_{s}=\left(T_{\mathrm{e}} m_{\mathrm{i}}\right)^{1 / 2} / e B_{0}$ to the geometric mean of the blobs cross-field size and the major radius. The drift advection terms are written using the Poisson bracket formalism $\{f, g\}=\partial_{x}\left(f \partial_{y} g\right)-$ $\partial_{y}\left(f \partial_{x} g\right)$. Typical scrape-off layer parameters are chosen by setting $R_{0}=1 \mathrm{~m}, B_{0}=1 \mathrm{~T}, T_{\mathrm{e}}=10 \mathrm{eV}$, and a typical blob cross-field size $\ell=1 \mathrm{~cm}$, which yields the dimensionless parameters $\delta=4.6 \times 10^{-3}$ and $\kappa=10^{-2}$. We choose $\nu=10^{-3}$ such that dissipation is much smaller than effective buoyancy [15]. The respective simulations are labeled as no compression $\alpha=\beta=0$, no electric drift compression $\alpha=0, \beta=1$, no diamagnetic drift compression $\alpha=1, \beta=0$, as well as full compression $\alpha=\beta=1$ throughout the rest of this contribution.

Equations 15 were solved with initial conditions given by Eqs. 3 using a spectral Fourier-Galerkin method to discretize spatial derivatives [46], as well as by discontinuous Galerkin methods (cf. FELTOR library [47]) for comparison. The detailed numerical codes including the input parameters as well as all output data can be found in the supplemental data to this contribution [48]. The results of the simulations were tested for convergence by increasing the domain size together with the number of cells and discretization points, as well as by reducing the diffusion coefficient, until no change in the blob dynamics was observable. The energy equations Eqs. 4 were verified numerically and we found negligible differences between the discontinuous and the Fourier-Galerkin methods.

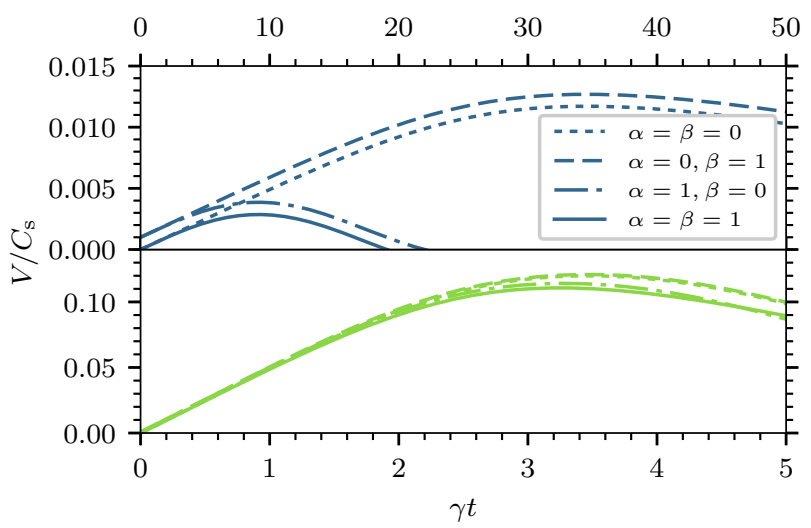

FIG. 1. (Color)The radial center of mass velocity of a blob with $\triangle n / N=0.02$ (upper panel) and $\Delta n / N=2$ (lower panel). An offset of $10^{-3}$ is added to the dashed and dasheddotted line for visibility.

Figure 1 shows the center of mass velocity 15 and 38 of the blob as a function of time for $\Delta n / N=0.02$ and $\Delta n / N=2$. In the case of a small initial amplitude, $\Delta n / N=0.02$, the blobs center of mass velocity initially increases approximately linearly in time for all four simulated cases. When neglecting electric drift compression, $\alpha=0$, the blob assumes a maximal radial velocity, $\max V \approx 1.2 \times 10^{-2} C_{\mathrm{s}}$ at $t_{\max V} \approx 32 \gamma^{-1}$. Including electric drift compression, $\alpha=1$, shortens the period of uniform acceleration. In this case the blob assumes a maximal radial center of mass velocity of $\max V \approx 3 \times 10^{-3} C_{\mathrm{s}}$ at $t_{\max V} \approx 10 \gamma^{-1}$. After this initial acceleration phase the blob decelerates and shows dispersion due to nonlinear mixing [15 and 19].

For $\Delta n / N=2$ the blob dynamics is independent of the included compressional terms in the model equations. After an approximately uniform acceleration phase the blob assumes a maximal radial velocity of $\max V \approx$ $0.11 C_{\mathrm{s}}$ at $t_{\max V} \approx 3 \gamma^{-1}$. We conclude that the electric drift compression has a profound influence on the dynamics of the blob in the case of small initial blob amplitudes.

Physically, the compressibility of the electric drift arises from the inhomogeneity of the magnetic field. The effect of including this term in the model equations is visualized in Fig. 2. We show the evolution of a blob with $\triangle n / N=0.02$ taken from simulations with $\alpha=\beta=0$ at $t=10 \gamma^{-1}$ in the upper column and at $t=35 \gamma^{-1}$ for 


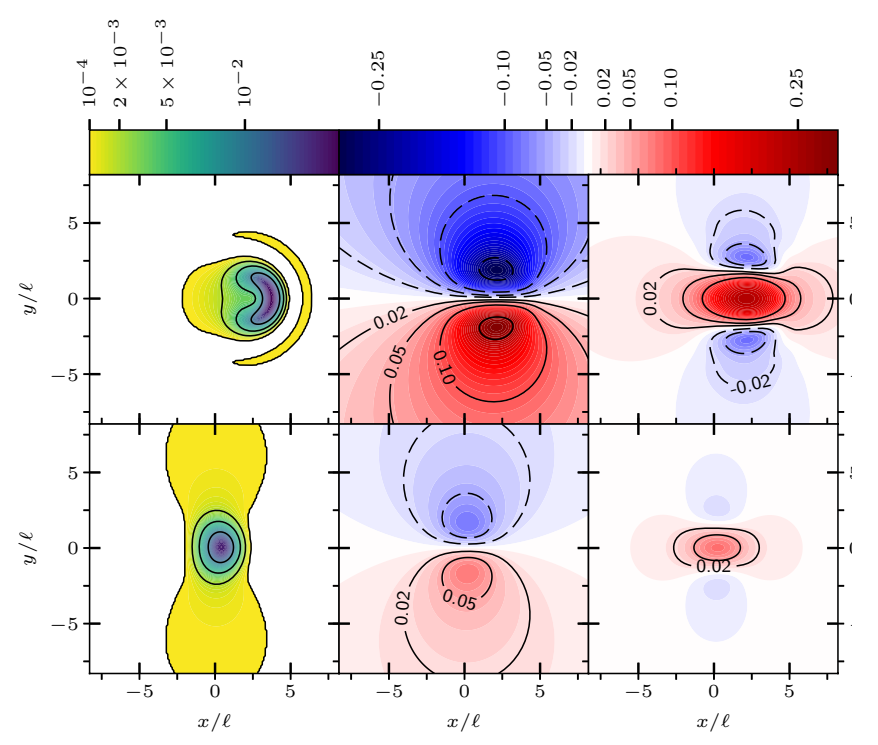

FIG. 2. (Color) Contour plots of the particle density perturbation (left column), the electrostatic potential (middle column) and the radial electric drift component (right column) for simulations without drift compression (upper row) and including drift compression terms (lower row). The initial amplitude is given by $\triangle n / N=0.02$ and the fields are shown at the time each blob propagates approximately at its maximal radial velocity, $t=35 \gamma^{-1}$ in the upper row and $t=10 \gamma^{-1}$ in the lower row. The black lines denote equi-density and equi-potential surfaces and correspond to the ticks in the colorbars.

$\alpha=1, \beta=0$ in the lower column. These are the times at which the blobs radial center of mass velocity is approximately maximal. The left column shows the particle density and the middle column the electric potential, where equi-potential lines give the flow field on which plasma is advected by the electric drift. In the right column we present the radial component of the electric drift, $-\partial \phi / \partial y$, which corresponds to the compression of the electric drift via $\nabla \cdot \boldsymbol{u}_{\mathrm{E}}=-(\partial \phi / \partial y) / B_{0} R_{0}$. Recall that this contribution is neglected in the density dynamics for $\alpha=0$ in the upper row. In both cases the flow field advects the blob radially outwards by transporting plasma from the front of the blob along the equi-potential lines poloidally above and below its density center into the wake of the blob. A finite electric drift compressibility inhibits this transport along the poloidal flanks. This leads to a poloidal elongation of the blob, as suggested in the lower left panel of the figure, and eventually to a dispersion of the density into two poloidally separated structures.

Figure 3 presents the blobs maximal radial center of mass velocity as a function of its initial perturbation amplitude. When drift compression is absent, $\alpha=\beta=0$, the maximal radial center of mass velocity of the blob is found to be proportional to the square root of its initial amplitude. This is in agreement with Eq. 6] From

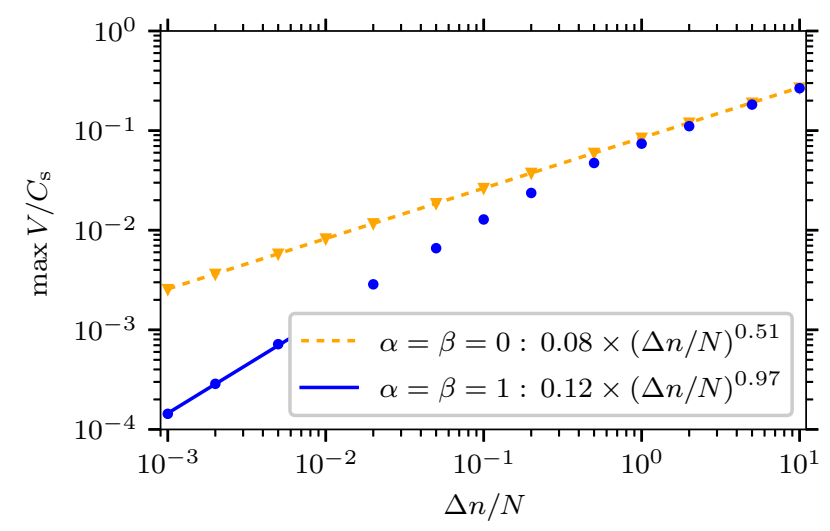

FIG. 3. Maximal radial center of mass velocity as a function of initial amplitude for $\kappa=10^{-2}$. The dashed and full line indicate a least squares fit to the maximal velocity for $\triangle n / N \leq 10^{-2}$.

a least squares fit of a power law to the simulation data we evaluate $\mathcal{R} \approx 0.85$. This is in agreement with previous studies of blob motion where a similar numerical value of $\mathcal{R}$ was found in the limit of negligible diffusion $[15$ and 19]. On the other hand, for small amplitudes the blobs radial velocity depends linearly on its initial amplitude when incorporating drift compression terms in the density dynamics. A least squares fit of a power law for $\triangle n / N \leq 10^{-2}$ yields $\mathcal{P} \approx 0.50$

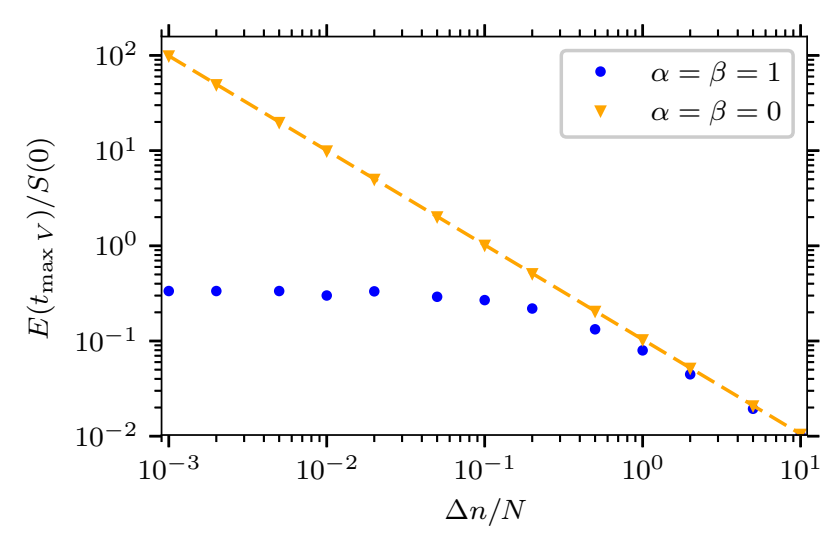

FIG. 4. (Color) Ratio of the kinetic energy at the time the blob is propagating at its maximal radial velocity to the initial value of $S$. The dashed line indicates a least squares fit of a power law to the simulation data with an exponent given by -1 .

Figure 4 shows the kinetic energy normalized to the initial value of the entropy at the time $t_{\max V}$ when the blob propagates at maximal radial velocity. Neglecting drift compression, maxima of this energy are up to two orders of magnitude larger than in simulations including drift compression for $\triangle n / N \ll 1$. For larger initial amplitudes the kinetic energy of the blob when traveling at maximum center of mass velocity approaches values found in simulations of the model including drift com- 
pression. A power law fit to the simulation data suggests that the relative kinetic energy is inversely proportional to $\triangle n / N$. When electric drift compression is included in the model the kinetic energy is bounded by the initial free energy $S(0)$. For amplitudes $\triangle n / N \lesssim 0.5$ approximately one third of the initial free energy is converted to kinetic energy.

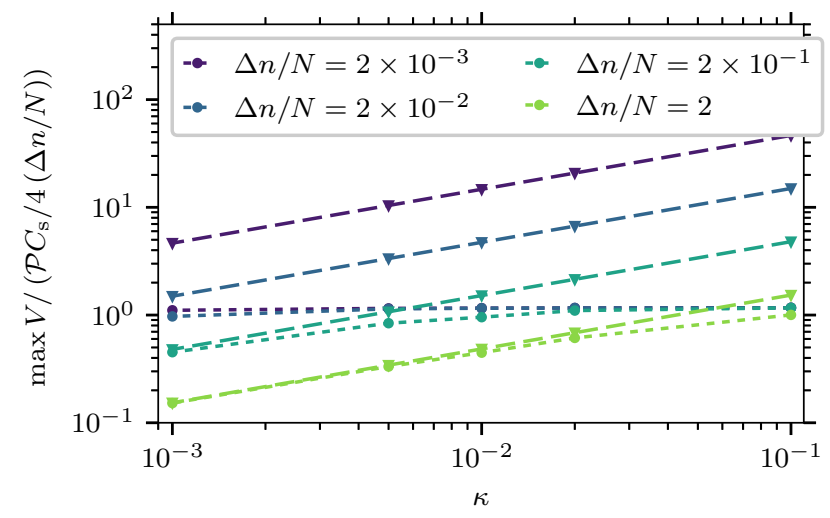

FIG. 5. (Color) Maximal radial center of mass velocity relative to the scaling given by Eq. 10 as a function of the blob cross-field size $\kappa$. The circles refer to simulation data of the compressional model, $\alpha=\beta=1$, and the triangles marks simulation data with $\alpha=\beta=0$.

Comparing the radial center of mass velocity as a function of the blobs cross-field size, shown in Fig. 5 reveals the different size scaling of the velocity in the compressible and incompressible cases. For the incompressible case, marked by the triangles, the radial center of mass velocity shows a square root dependence on the blobs cross-field size, as suggested by the inertial scaling of Eq. 6. When compression is included, the velocity is independent of the cross-field size for small initial amplitudes. For sufficiently large amplitudes, the square root scaling with blob size is observed also for the compressible case in Fig. 5. In agreement with Eq. 11, the numerical simulations of the compressible model show that the transition indeed depends on both the cross-field size and the amplitude.

The maximal radial center of mass velocities as a function of initial amplitude for various cross-field sizes are shown in Fig. 6. For small amplitudes, $\triangle n / N \lesssim 10^{-2}$, the maximal radial velocity becomes independent of the blobs cross-field size and depends approximately linearly on its initial amplitude. For larger initial amplitudes the maximal velocity transitions into the square root dependence on the blobs initial amplitude. Furthermore, max $V$ depends on $\kappa$ for large $\triangle n / N$ as also seen in Fig. 5. This dependence is in excellent agreement with the predictions from Eqs. 6] and [10.

The numerical simulations further demonstrate that the time at which the blob assumes its maximal radial center of mass velocity becomes independent of its initial amplitude in the limit of small initial amplitudes,

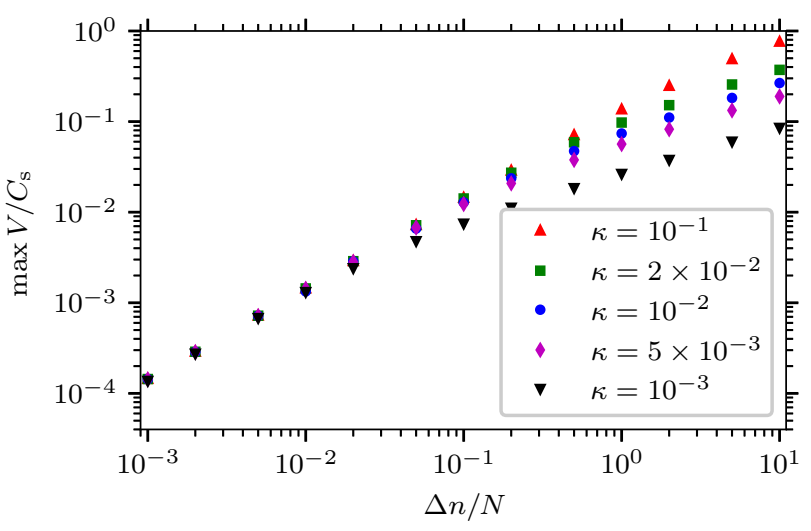

FIG. 6. (Color) Maximal radial center of mass velocity as a function of its initial amplitude for varying ratios of the blob cross-field size to major radius.

as shown in Fig. 7. This is in agreement with Eq. 14. The range over which $t_{\max V}$ is independent of $\triangle n / N$, is consistent with the critical initial amplitude Eq. 11 .

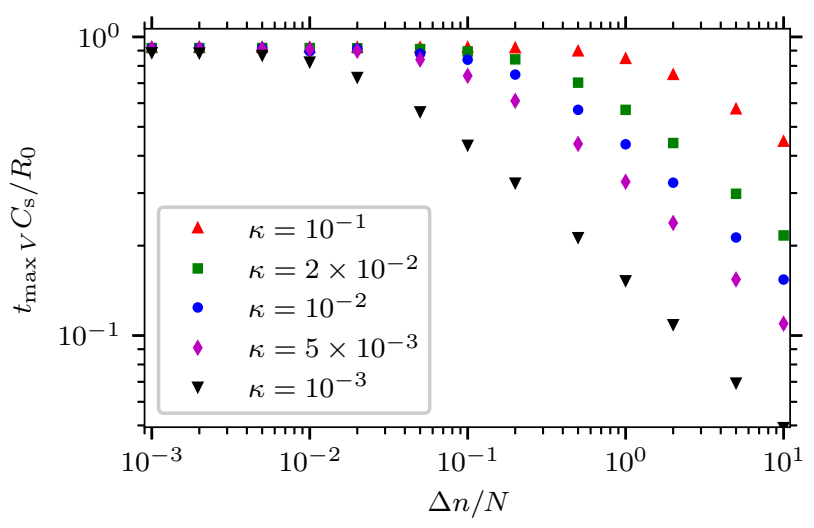

FIG. 7. (Color) Time at which the blob propagates at maximal radial center of mass velocity as a function of its initial amplitude.

The simulations show furthermore that all blobs feature an initial period of constant acceleration with the acceleration proportional to its initial amplitude and independent of its cross-field size. This is clearly seen in Fig. 8 and in agreement with Eq. 13. This corroborates the basis of the scale analysis leading to Eq. 6. namely that initially the blob is subject to uniform acceleration by an effective gravity, given by the interchange term in Eq. 2b.

The break in slopes for fixed $\kappa$ in the simulation data shown in Figs. [6] and 7 is predicted to appear at an amplitude given by Eq. [11, Earlier in this section we obtained $\mathcal{P} \approx 0.50$ by a least squares fit of Eq. 10 to simulation data for the compressible case with $\kappa=10^{-2}$. A least squares fit of Eq. 6] to simulation data for the incompressible flow model gives $\mathcal{R} \approx 0.85$, such that $(\triangle n / N)_{\mathrm{c}} \approx 46 \kappa$. We continue by comparing this pre- 


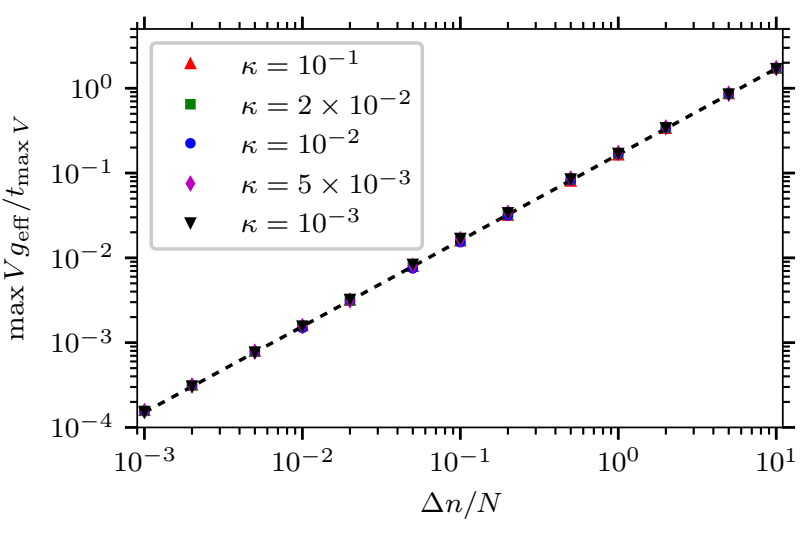

FIG. 8. (Color) Maximal radial center of mass velocity of the blob divided by the time at which the blob assumes this velocity. The dashed line denotes a power law fit with exponent 1.0 from which we evaluate $\mathcal{Q} \approx 0.34$.

dicted value to the simulation data.

To this end we fit Eqs. 6] and 10 to the simulation data shown in Fig. 6, as well as Eq. 14 to the simulation data shown in Fig. 7. The fit range is chosen to be the range where the data points approximately follow a power law scaling and the estimated transition density amplitude is given by the intersection of the extrapolated fits. Fig. 9 compares the transition amplitude estimated by intersection of fits to the velocity data (green squares) and fits to the $t_{\max V}$ data (red pentagon) to Eq. 11] (black circles).

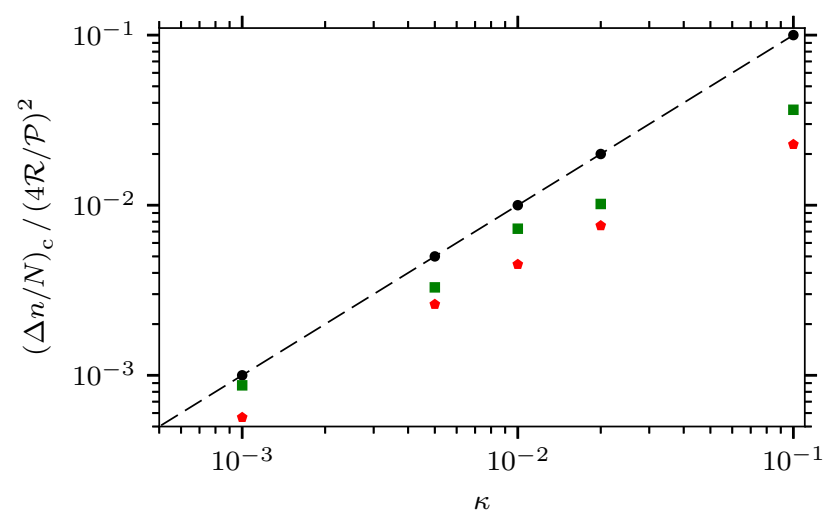

FIG. 9. (Color) The critical transition amplitude Eq. 11 evaluated with $\mathcal{P}=0.50$ and $\mathcal{R}=0.85$ (black circles) compared to estimates of the transition amplitude from the velocity parameter scan (green squares) and the corresponding times at which the blob propagates at its maximal radial velocity (red pentagon).

For small blob cross-field sizes, $\kappa=10^{-3}$, the intersection of the fits to the velocity data yields $(\triangle n / N)_{\mathrm{c}} \approx$ $4.0 \times 10^{-2}$, the transition of the fits to the $t_{\max V}$ data yields $(\triangle n / N)_{\mathrm{c}} \approx 2.5 \times 10^{-2}$ while Eq. 11 yields $(\triangle n / N)_{\mathrm{c}} \approx 4.6 \times 10^{-2}$. The discrepancy between the estimates increases with $\kappa$ as the amplitude range over which the simulation data follows an exact power law de- creases. For $\kappa=10^{-1}$ the intersection of the fits to the $\max V$ data yields $(\triangle n / N)_{\mathrm{c}} \approx 1.7$, the intersection of fits to the $t_{\max V}$ data yields $(\Delta n / N)_{\mathrm{c}} \approx 1.0$ while Eq. 11 evaluates to approximately 4.6 . These values give a relative error of $0.61(0.78)$ on the transition amplitude, given by Eq. 11, when compared to the $\max V\left(t_{\max V}\right)$ data.

\section{DISCUSSION AND CONCLUSIONS}

In conclusion, we have analyzed a two-field fluid model commonly used to describe blob dynamics in the scrapeoff layer of magnetically confined plasmas, in basic laboratory plasma experiments and irregularities in ionospheres of celestial bodies. In section 2 we discussed a commonly employed simplification, namely neglecting the compression of the electric and diamagnetic drift in the particle continuity equation, and showed that neglecting the electric drift compression corresponds to reducing the number of conservation laws of the system. In the compressible case the initialization of the density field introduces an additional constraint on the energy conservation. This introduces an upper limit on the blobs radial center of mass velocity for small amplitudes and suggests that a velocity scaling regime exists where the blobs center of mass velocity depends linearly on its initial perturbation amplitude and is independent on its cross-field size, $V \sim \triangle n / N$.

For this linear scaling, the time it takes a blob to assume its maximal radial velocity is independent of its cross-field size or amplitude. A scale analysis of the model equations suggests on the other hand that the center of mass velocity scales with the square root of the blobs initial amplitude and cross-field size. For this velocity scaling, $t_{\max V}$ is proportional to $\left(\ell / R_{0}\right)^{1 / 2}$ as well as to $(\triangle n / N)^{-1 / 2}$. Assuming a smooth transition between these two velocity scalings in the compressible model we find that the transition point depends on both, the initial amplitude of the blob and its cross-field size. For the incompressible model, the cross-field size may be absorbed in the normalization of the model [15] and only the square root velocity scaling is expected to hold.

In section 3 we presented numerical simulations of seeded blobs using the reduced model equations. Both scaling regimes are recovered and the transition between them is found to depend on the postulated parameters. We estimated the numerical parameters for the velocity scaling laws given by Eqs. 6] and Eq. 10, the transition amplitude given by Eq. 11 and the time at which the blob propagates at its maximal radial velocity given by Eq. 14 for a fixed initial blob cross-field size. The predicted transition point between the velocity scalings is shown to agree with the transition point found by fits to data from numerical simulations.

A large number of publications studying the dynamical properties of seeded blob structures in tokamak scrape-off layers employ models in which compressional 
effects in the particle density dynamics are neglected [14, 15, 19, 32, 33, 41, 50 52]. This is the first contribution to show that for typical blob cross-field sizes their dynamics is insensitive to electric drift compression only when the initial blob amplitude exceeds approximately half the background density. In this amplitude range the Boussinesq approximation used in the previous references and in this work is not strictly valid. Conclusively, we find that models incorporating an inhomogeneous magnetic field need to retain drift compression terms.

A direct application of velocity scaling laws for plasma filaments is to apply them to the problem of broad particle density profiles observed in the scrape-off layer of magnetically confined plasmas. A recently developed stochastic model predicts that the density profile is proportional to the average blob amplitude and the duration time in which a single blob traverses a given point $[6,12,24,53$, and 54]. On the other hand the duration time is given by the ratio of the blobs cross-field size to its radial velocity. In turn, this allows to refine the dependence of the particle density profile on blob properties and to test these predictions against experimental measurements.

Future work will study the robustness of the derived scalings by comparing them to more involved gyro-fluid simulations that relieve the Boussinesq approximation. It is further planned to elucidate the effect of energy conservation on blob dynamics in fluid models, which parameterize the parallel dynamics and include the effects of magnetic field lines intersecting material walls.

\section{ACKNOWLEDGEMENTS}

We would like to acknowledge fruitful discussions with M. Held. The authors were supported with financial subvention from the Research Council of Norway under grant 240510/F20. M.W. was supported by the Austrian Science Fund (FWF) Y398. The computational results presented have been achieved in part using the Vienna Scientific Cluster (VSC) and by the Norwegian meta center for computational science (NOTUR) program through grants of computation time
[1] S. J. Zweben, Phys. Fluids 28974 (1985).

[2] G. Y. Antar, S. I. Krasheninnikov, P. Devynck, R. P. Doerner, E. M. Hollmann, J. A. Boedo, S. C. Luckhardt and R. W. Conn, Phys. Rev. Lett 87065001 (2001); G. Y. Antar, P. Devynck, X. Garbet and S. C. Luckhardt, Phys. Plasmas 81612 (2001); G. Y. Antar, G. Counsell, Y. Yu, B. LaBombard and P. Devynck, Phys. Plasmas 10419 (2003) and references therein.

[3] J. A. Boedo, D. L. Rudakov, R. J. Colchin, R. A. Moyer, S. Krasheninnikov, D. G. Whyte, G. R. McKee, G. Porter, M. J. Schaffer, P. C. Stangeby, W. P. West, S. L. Allen, and A. W. Leonard, Journ. Nucl. Mater. 313316813 (2003).

[4] J. L. Terry, S. J. Zweben, K. Hallatscheck, B. LaBombard, R. J. Maqueda, B. Bai, C. J. Boswell, M. Greenwald, D. Kopon, W. M. Nevins, C. S. Pitcher, B. N. Rogers, D. P. Stotler, and X. Q. Xu, Phys. Plasmas 10 1739 (2003).

[5] M. Agostini, S. J. Zweben, R. Cavazzana, P. Scarin, G. Serianni, R. J. Maqueda, and D. P. Stotler, Phys. Plasmas 14102305 (2007).

[6] O. E. Garcia, S. M. Fritzner, R. Kube, I. Cziegler, B. LaBombard, and J. L. Terry, Phys. Plasmas 20055901 (2013).

[7] M. Umansky, S. I. Krasheninnikov, B. LaBombard and J. L. Terry, Phys. Plasmas 53373 (1998).

[8] B. Lipschultz, B. LaBombard, C. S. Pitcher, and R. Boivin, Plasma Phys. Control. Fusion 44733 (2002).

[9] B. LaBombard, R. L. Boivin, M. Greenwald, J. Hughes, B. Lipschultz, D. Mossessian, C. S. Pitcher, J. L. Terry, S. J. Zweben,b) and Alcator Group, Phys. Plasmas 82107 (2001).

[10] D. Carralero, P. Manz, L. Aho-Mantila, G. Birkenmeier, M. Brix, M. Groth, H. W. Müller, U. Stroth, N. Vianello, E. Wolfrum, ASDEX Upgrade team, JET Contributors, and EUROfusion MST1 Team, Phys. Rev. Letters 115 215002 (2015); D. Carralero, G. Birkenmeier, H. W. Müller, P. Manz, P. deMarne, S. H. Müller, F. Reimold, U. Stroth, M. Wischmeier, E. Wolfrum and The ASDEX Upgrade Team, Nucl. Fusion 54123005 (2014).

[11] R. Kube, A. Theodorsen, O. E. Garcia, B. LaBombard and J. L. Terry, Plasma Phys. Control. Fusion 58054001 (2016).

[12] O. E. Garcia, R. Kube, A. Theodorsen, and H. L. Pécseli, Phys. Plasmas 23052308 (2016).

[13] D. A. D'Ippolito, J. R. Myra, and S. I. Krasheninnikov, Phys. Plasmas 9222 (2002).

[14] N. Bian, S. Benkadda, J. V. Paulsen, and O. E. Garcia, Phys. Plasmas 10671 (2003).

[15] O. E. Garcia, N. H. Bian, V. Naulin, A. H. Nielsen, and J. Juul Rasmussen, Phys. Plasmas 12090701 (2005).

[16] S. I. Krasheninnikov, Phys. Lett. A 283368 (2001).

[17] D. A. D’Ippolito, J. R. Myra, S. I. Krasheninnikov, G. Q. Yu, and A. Yu. Pigarov, Contrib. Plasma Phys. 44205 (2004).

[18] D. A. D'Ippolito, J. R. Myra and S. J. Zweben, Phys. Plasmas 18060501 (2011).

[19] O. E. Garcia, N. H. Bian, W. Fundamenski, Phys. Plasmas 13082309 (2006).

[20] O. E. Garcia, N. H. Bian, V. Naulin, A. H. Nielsen and J. Juul Rasmussen, Phys. Scr. T122 104 (2006).

[21] D. L. Rudakov, J. A. Boedo, R. A. Moyer, P. C. Stangeby, J. G. Watkins, D. G. Whyte, L. Zeng, N. H. Brooks, R. P. Doerner, T. E. Evans, M. E. Fenstermacher, M. Groth, E. M. Hollmann, S. I. Krasheninnikov, C. J. Lasnier, A. W. Leonard, M. A. Mahdav, G. R. McKee, A. G. McLean, A. Yu. Pigarov, W. R. Wampler, G. Wang, W. P. West and C. P. C. Wong, Nucl. Fusion 451589 (2005).

[22] O. Grulke, J. L. Terry, B. Labombard, and S. J. Zweben, Phys. Plasmas 13012306 (2006). 
[23] O. Grulke, J. L. Terry, I. Cziegler, B. LaBombard and O. E. Garcia, Nucl. Fusion 54043012 (2014).

[24] A. Theodorsen, O. E. Garcia, J. Horacek, R. Kube, and R. A. Pitts, Plasma Phys. Control. Fusion 58044006 (2016).

[25] N. Katz, J. Egedal, W. Fox, A. Le, and M. Porkolab, Phys. Rev. Lett. 101015003 (2008).

[26] C. Theiler, I. Furno, P. Ricci, A. Fasoli, B. Labit, S.H. Müller, and G. Plyushchev, Phys. Rev. Lett. 103 (2009).

[27] I. Furno, C. Theiler, D. Lançon, A. Fasoli, D. Iraji, P. Ricci, M. Spolaore and N. Vianello, Plasma Phys. Control. Fusion 53124016 (2011).

[28] F. Riva, C. Colin, J. Denis, L. Easy, I. Furno, J. Madsen, F. Militello, V. Naulin, A. H. Nielsen, J. M. B. Olsen, J. T. Omotani, J. Juul Rasmussen, P. Ricci, E. Serre, P. Tamain and C. Theiler, Plasma Phys. Control. Fusion 58 044005 (2016).

[29] D. L. Hysell, Journ. Atmos. Solar-Terr. Phys. 621037 (2000).

[30] A. M. Rymer, B. H. Mauk, T. W. Hill, N. Andre, D. G. Mitchell, C. Paranicas, D. T. Young, H. T. Smith, A. M. Persoon, J. D. Menietti, G. B. Hospodarsky, A. J. Coates, M. K. Dougherty, Planet. Space Science 571779 (2009).

[31] R. A. Wolf, C. X. Chen, and F. R. Toffoletto, Journ. Geophys. Res. 117 A02215 (2012).

[32] D. A. D'Ippolito and J. R. Myra, Phys. Plasmas 104029 (2003)

[33] J. R. Myra, D. A. D'Ippolito, S. I. Krasheninnikov and G. Q. Yu, Phys. Plasmas 114267 (2004).

[34] G. Q. Yu, S. I. Krasheninnikov, and P. N. Guzdar, Phys. Plasmas 13042508 (2006).

[35] J. Angus, and S. I. Krasheninnikov, Phys. Plasmas 21 112504 (2014)

[36] J. T. Omotani, F. Militello, L. Easy and N. R. Walkden, Plasma Phys. Control Fusion 58014030 (2015).

[37] M. Held, M. Wiesenberger, J. Madsen, and A. Kendl, Nucl. Fusion 56126005 (2016).
[38] M. Wiesenberger, J. Madsen, and A. Kendl, Phys. Plasmas 21092301 (2014).

[39] J. Madsen, O. E. Garcia, J. S. Larsen, V. Naulin, A. H. Nielsen, and J. Juul Rasmussen, Phys. Plasmas 18 112504 (2011).

[40] P. Manz, D. Carralero, G. Birkenmeier, H. W. Müller, S. H. Müller, G. Fuchert, B. D. Scott, and U. Stroth, Phys. Plasmas 20102307 (2013)

[41] R. Kube and O. E. Garcia, Phys. Plasmas 18102314 (2011); R. Kube and O. E. Garcia, Phys. Plasmas 19 042305 (2012).

[42] J. R. Myra and D. I. D'Ippolito, Phys. Plasmas 12092511 (2005).

[43] J. Olsen, J. Madsen, A. H. Nielsen, J. Juul Rasmussen and V. Naulin, Plasma Phys. Control. Fusion 58044011 (2015).

[44] E. Ott, J. Geophys. Res. 83, 2066, (1978)

[45] B. Scott, Phys. Plasmas 12102307 (2005).

[46] https://github.com/rkube/2dads

[47] M. Wiesenberger, Gyrofluid computations of filament dynamics in tokamak scrape-off layers, PhD thesis (2014), University of Innsbruck, Austria; https://github.com/feltor-dev/feltor

[48] Replication Data for: Amplitude scaling for interchange motions of plasma filaments http://dx.doi.org/10.18710/7RRESR

[49] S. H. Müller, C. Theiler, A. Fasoli, I. Furno, B. Labit, G. R. Tynan, M. Xu, Z. Yan and J. H. Yu, Plasma Phys. Control. Fusion 51055020 (2009).

[50] J. R. Angus, S. I. Krasheninnikov, and M. V. Umansky, Phys. Plasmas 19082312 (2012).

[51] S. I. Krasheninnikov and S. I. Smolyakov, Phys. Plasmas 15055909 (2008).

[52] J. R. Myra, D. A. Russel, and D. A. D'Ippolito, Phys. Plasmas 13112502 (2006).

[53] O. E. Garcia, Phys. Rev. Letters 108265001 (2012).

[54] R. Kube and O. E. Garcia, Phys. Plasmas 22012502 (2014). 